\title{
Laryngeal Amyloidosis: A Case Report
}

\author{
Raja Salman • M. Lateef • Irfan Iqbal • \\ Ayaz Rehman $\cdot$ Mudasir ul Islam
}

Received: 7 November 2008/ Accepted: 23 February 2009/Published online: 13 January 2011

(C) Association of Otolaryngologists of India 2011

\begin{abstract}
We report a case of Amyloidosis of larynx in a 60-year-old adult who reported to us with hoarseness of voice of 3 years long duration and no other symptoms. Patient was treated successfully with endoscopic curettage of the mass with no inadvertent ill effects.
\end{abstract}

\section{Introduction}

Amyloid is a heterogeneous family of extracellular proteinaceous deposits with characteristic microscopic, histochemical, and ultrastructural features. Deposits of amyloid in the larynx are rare, accounting for between 0.2 and $1.2 \%$ of benign tumors of the larynx. Amyloid in the larynx can be identified as subepithelial extracellular deposits of acellular, homogeneous and amorphous, eosinophilic material displaying apple-green birefringence with polarized light when stained with Congo red or that is metachromatic with crystal violet or methyl violet.

Amyloidosis can affect virtually any organ or tissue in the body. Macroglossia is the most common head and neck manifestation of amyloidosis, and appears in between 15 and $20 \%$ of patients with light chain disease. The laryngotracheal system is the next most common site. Amyloidosis constitutes approximately $1 \%$ of all benign lesions of the larynx. It usually occurs as nodules or polypoid lesions and can be located anywhere in the larynx or trachea. Systemic involvement is very rare with laryngeal amyloidosis (Fig. 1a, b).

R. Salman $(\bowtie) \cdot$ M. Lateef $\cdot$ I. Iqbal · A. Rehman · M. ul Islam Deparment of ENT, HNS, Government Medical College,

Srinagar, J \& K, India

e-mail: dr_raja_salman@yahoo.co.in

\section{Case Report}

A 60 years old healthy looking adult male hypertensive for last 3 years on treatment, non-smoker, non-diabetic, was brought to our out-patient department with a history of hoarseness of voice for the last 3 years. There was no associated breathing difficulty, or any history of painful deglutition or swallowing. A contrast enhanced CT scan of the patient shows smooth mass involving supraglottic region. A biopsy of the supraglottic mass showed evidence of amyloidosis which was later confirmed by immunohistochemical studies. On second surgery the mass had regressed in size and the left over tissue was removed by endoscopic curettage. Post-op stay in hospital was uneventful and on subsequent follow-up the voice had considerably improved. Patient is doing well at 6 months post-op.

\section{Discussion}

Amyloidosis is typified by deposition of insoluble proteinaceous material which has typical staining properties and electron microscopic appearance. Its hallmark is an apple green birefringence under polarized microscopy. Under the electron microscope, amyloid appears as a mass of rigid, non-branching fibrils. X-ray crystallography reveals that these fibrils have a regular, antiparallel, betapleated sheet configuration.

Different sources of amyloidosis are recognized and include [1] immunoglobulin light chains, [2] amyloid A in reactive amyloidosis, [3] transthyretin type in familial or senile amyloid, and [4] hemodialysis-associated amyloid. The literature supports that most cases of amyloidosis of the larynx are composed of a protein that is 
Fig. 1 a, b Soft tissue mass involving supraglottic region with tissue biopsy and further evaluation revealing laryngeal amyloidosis
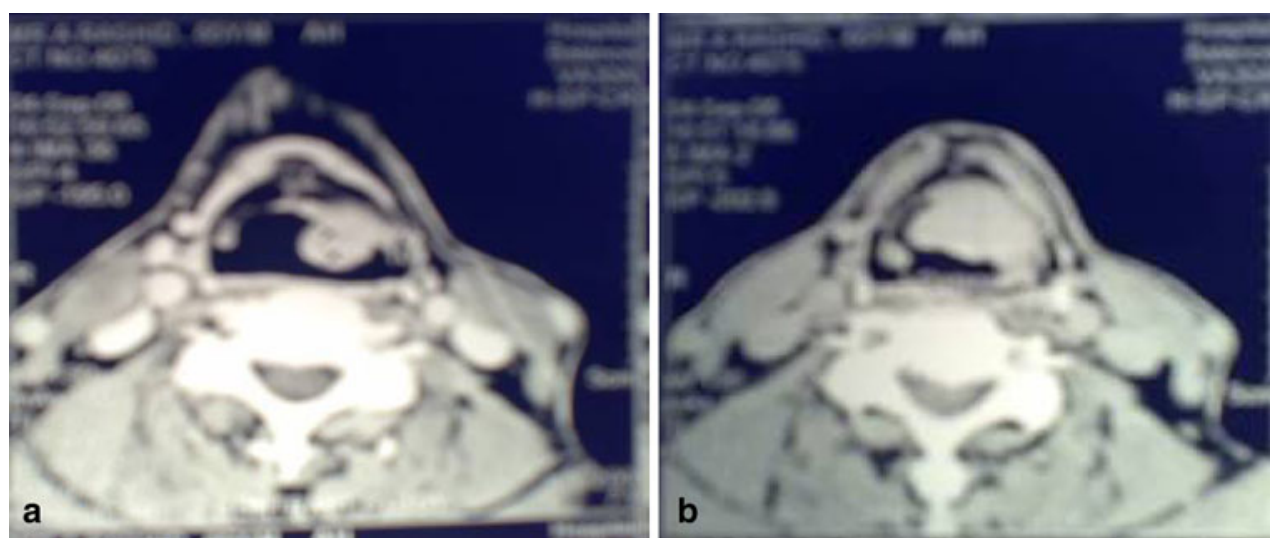

immunologically identical to the variable region of the light chain fragment of immunoglobulin and is classified as a fibril type. Although immunophenotyping has become universally available, the "gold standard" for the diagnosis of amyloid remains a tissue biopsy demonstrating characteristic hematoxylin and eosin changes and Congo red birefringence or metachromatic pink-violet staining with methyl violet or crystal violet The immunoglobulin nature of laryngeal amyloid is accepted, but the source of the immunoglobulin is unclear. The lesion may develop from a localized monoclonal immunoproliferative disorder, in which the plasma cells, which are intimately associated with the amyloid deposits, are thought to produce the light chain immunoglobulin that is deposited as amyloid, rather than represent an inflammatory infiltrate reacting to the deposited amyloid This intimate association of the lymphoplasmacytic infiltration is different from systemic amyloidosis whereby the plasma cells are spatially separated from the amyloid deposition The second theory for the amyloid deposition suggests that a circulating precursor protein is deposited in the stroma after a change in the vascular permeability as a result of local inflammation.

Accurate diagnosis is often delayed up to a year or more. Diagnosis is usually initiated by clinical suspicion but this must be confirmed by histologic examination of tissue. Under some instances this may be obtained by biopsy of an obvious lesion. However, in the majority of cases, no easily accessible lesion is present. The gold standard for diagnosis of systemic amyloidosis in the past consisted of a rectal biopsy, which was felt to be positive in approximately $80 \%$ of patients. Recently, though, the preferred method has become simple needle biopsy of clinically uninvolved abdominal subcutaneous fat. This has the obvious advantage of being very safe and less uncomfortable to the patient. This method is positive in $95 \%$ of prim systemic, $66 \%$ of secondary systemic, and $86 \%$ of heredofamilial amyloidosis cases.

Since the vast majority of lesions of the head and neck, with the exception of macroglossia, represent localized disease, therapy consisting of surgical excision has been far more successful. In the larynx, a conservative approach has been recommended consisting of local excision using laser or microlaryngeal instruments with conservation of surrounding tissue. Recent literature has advocated $\mathrm{CO}_{2}$ laser as the treatment of choice. Recurrence is rare, and, if it occurs, can be treated with further conservative excision.

\section{References}

1. Ma L, Bandarchi B, Sasaki C (2005) Primary localized laryngeal amyloidosis: report of 3 cases with long-term follow-up and review of the literature. Arch Pathol Lab Med 129(2):215-218

2. Lewis JE, Olsen KD, Kurtin PJ, Kyle RA (1992) Laryngeal amyloidosis: a clinicopathologic and immunohistochemical review. Otolaryngol Head Neck Surg 106:372-377

3. Behranwala KA, Ali Asgar B, Borges Anita (2004) Laser in treatment of laryngeal amyloidosis. Indian J Otolaryngol Head Neck Surg 56(1):46-48

4. Mittrani M, Biller HF (1985) Laryngeal amyloidosis. Laryngoscope 95:1346-1347

5. Andrews AH Jr, Polanyi TG, Grybauokas VT (1983) General and clinical considerations in laryngologic laser. Otolaryngol Clin North Am 16:793-806 